\title{
Stochastic seismic anisotropy sequential inversion for geomechanical properties and in-situ stress estimation
}

Uilli Freitas, Igor Braga, Pedro Canhaço and Renzo Francia, Invision Geophysics, Brazil

Copyright 2019, SBGf - Sociedade Brasileira de Geofísica

This paper was prepared for presentation during the $16^{\text {th }}$ International Congress of the Brazilian Geophysical Society held in Rio de Janeiro, Brazil, 19-22 August 2019.

Contents of this paper were reviewed by the Technical Committee of the $16^{\text {th }}$ International Congress of the Brazilian Geophysical Society and do not necessarily represent any position of the SBGf, its officers or members. Electronic reproduction or storage of any part of this paper for commercial purposes without the written consent of the Brazilian Geophysical Society is prohibited.

\section{Abstract}

Seismic anisotropy inversion has been principally used to infer anisotropic characteristics of the reservoir, such as fracture orientation and intensity. However, it is also possible to obtain geomechanical properties and in-situ stresses when we extend this approach. For it, mathematical simplified poroelastic models can be used incorporating the relationship among geomechanical properties, rock strength, and anisotropic parameters. Thus, we present a stochastic seismic anisotropy sequential inversion of $P$-wave pre-stack azimuthal data for geomechanical properties and in-situ stress estimation. We consider an HTI media, whose reflection coefficients are calculated by Rüger approximation and use the Linear Slip Theory (LST) to achieve a poroelastic model aiming to relate anisotropic parameters to stress. We apply the stochastic inversion in a 1D synthetic data demonstrating the applicability of the proposed inversion methodology.

\section{Introduction}

It is well known that seismic analysis and inversion are one of the most relevant and useful methods to estimate characteristics and parameters of the reservoir. From these methods, the analysis of amplitude versus offset/angle (AVO/AVA) gained importance with works published by Richards and Frasier (1976), Ostrander (1984), Shuey (1985), etc. However, the usability of this approach is restrictive, because the theory behind it is based on analytic expressions for $\mathrm{P}$-wave reflection coefficients in isotropic media (Rüger, 1997).

Works have been discussing about the importance in considering the anisotropy effects in the seismic data signature and, in the AVO analysis, it was shown that the seismic response may be considerably distinctive from the 'conventional' one. Nevertheless, only with the refined Pwave reflection coefficients approximations, proposed by Rüger (1997), it was possible to outstanding how the anisotropic parameters (Thomsen's parameters) influences in the AVO signature in a media which the properties change azimuthally.

To the azimuthal anisotropy media, Rüger (1997), following Thomsen (1993), derived a linearized approximation for P-
$P$ reflection coefficient using a transversely isotropic model with a horizontal axis of rotational symmetry $(\mathrm{HTI})$. This model is frequently used to describe the effect of natural fracturing and in-situ stress on the elastic behavior of rocks (Mavko et al., 1995; Gray et al., 2012). The amplitude variation with angle and azimuth (AVAz) inversion is mainly based on this approximation.

AVAz inversion has been largely used to estimate fracture orientation and intensity (Willian and Jenner, 2002; Mahmoudian and Margrave, 2012), but more relevant parameters - fracture compliances and in-situ stress can be obtained when a physical model is applied, generally the Linear Slip Theory (LST). Gray et al., (2012) demonstrate this methodology applying it in a shale play, which the main objective was to optimize the placement and direction of horizontal wells and hydraulic fracture stimulations. Another important applicability of in-situ stress prior knowledge is the construction and validation of 3D Mechanical Earth Model (3D MEM), improving wellbore stability procedures as well as mitigating different types of geohazards during the oil and gas field exploitation.

As mentioned, AVAz inversion is determinant to estimation of the properties described above. For it, different methods have been tested, Rüger Azimuthal AVO inversion, Azimuthal Fourier Coefficients, Azimuthal Fourier Coefficients Elastic Inversion, and each one has advantages and disadvantages, for example: ambiguity or unambiguity in the anisotropy direction, the type of property estimated (layer or interface) and the resolution (Delbecq et al., 2013).

Thus, in this work, we develop a stochastic seismic anisotropy sequential inversion of $\mathrm{P}$-wave pre-stack azimuthal data for geomechanical and in-situ stress estimation, incorporating the advantages of the distinctive methods seen in the literature. To demonstrate the applicability of the proposed inversion methodology we test it in a 1D synthetic data. The results show that was possible to solve the ambiguity of the anisotropy direction and obtain reliable values to the estimated parameters.

\section{$\mathrm{P}$-wave reflection in $\mathrm{HTI}$ media}

$\mathrm{HTI}$ media is characterized by a horizontal axis of rotational symmetry and has two vertical symmetry planes: the plane formed by the symmetry axis, called symmetry-axis plane, and the plane perpendicular to the symmetry axis, called isotropy plane (Fig. 1). When the HTI symmetry is caused by a set of vertical fractures, the isotropy plane coincides with the fracture plane. 


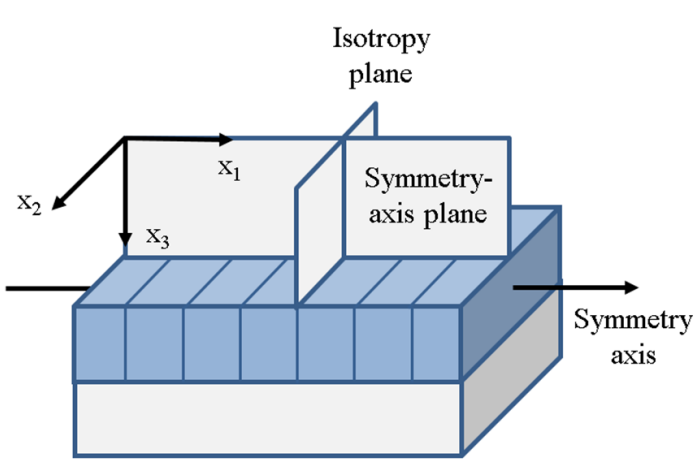

Figure 1: HTI model representation showing two vertical planes, the symmetry-axis plane and the isotropy plane.

According Rüger (1997), the PP reflection coefficient at a boundary between two HTI media with the same orientation of the symmetry-axis plane and symmetry axis along $\phi_{0}$ azimuth can be expressed in function of the incidence angle $i$ and source-receiver azimuth $\phi$ :

$R_{P P}(i, \phi)=\frac{1}{2} \frac{\Delta Z}{\bar{Z}}+\frac{1}{2}\left\{\frac{\Delta \alpha}{\bar{\alpha}}-\left(\frac{2 \bar{\beta}}{\bar{\alpha}}\right)^{2} \frac{\Delta G}{\bar{G}}+\left[\Delta \delta^{(V)}+\right.\right.$

$\left.\left.2\left(\frac{2 \bar{\beta}}{\bar{\alpha}}\right)^{2} \Delta \gamma\right] \cos ^{2}\left(\phi-\phi_{0}\right)\right\} \sin ^{2} i+\frac{1}{2}\left\{\frac{\Delta \alpha}{\bar{\alpha}}+\Delta \epsilon^{(V)} \cos ^{4}(\phi-\right.$ $\left.\phi_{0}\right)+\Delta \delta^{(V)} \sin ^{2}\left(\phi-\phi_{0}\right) \cos ^{2}(\phi-$

$\left.\left.\phi_{0}\right)\right\} \sin ^{2} i \tan ^{2} i$,

where the overstrike indicates an average and $\Delta$ denotes the difference in the properties across the boundary. The parameters $\beta$ and $\alpha$ represents the isotropy-plane velocities of the fast vertical shear-wave (polarized parallel to de isotropy plane) and the vertical compressional wave, respectively. The parameter $\rho$ is the bulk density, $G$ the vertical shear modulus $\left(G=\rho \beta^{2}\right)$ and $Z$ the vertical Pwave impedance $(Z=\rho \alpha), \epsilon^{(V)}, \quad \delta^{(V)}$ denote the anisotropic coefficients introduced by Rüger (1997), where the subscript (V) symbolize the symmetric analogy assumption with Thomsen's (1986) parameters for VTI media and $\gamma$ corresponds to the generic Thomsen's parameter defined with respect to the horizontal symmetry axis.

\section{Fracture orientation}

For fracture orientation estimation we use the equation (1) considering incident angle data with less than $35^{\circ}$, thus the last term can be excluded and equation (1) is rewritten as:

$$
R_{P P}(i, \phi)=I+Q \sin ^{2} i,
$$

in this equation $I$ is the AVO intercept and $Q$ is the gradient, which is in function of an isotropic gradient $G_{i s o}$, an anisotropic gradient $G_{a n i}$ and $\phi_{0}$ :

$$
R_{P P}(i, \phi)=I+\left\{G_{\text {iso }}+G_{\text {ani }} \cos ^{2}\left(\phi-\phi_{0}\right)\right\} \sin ^{2} i .
$$

The usual approach to $\phi_{0}$ estimation is to linearize the equation (3), either by changing variables (Mahmoudian and Margrave, 2012) or by rewriting it in terms of $\cos (2 \phi)$ and $\sin (2 \phi)$ (Downton and Gray, 2006), then solving the linear inversion problem. The point is that the orientation estimated has a $90^{\circ}$ ambiguity. For this reason we propose a different way to recovery the fracture orientation. Firstly, we do a linear deterministic inversion for all azimuths, based on equation (2), obtaining different values of gradient $Q$ for each azimuth and same value of intercept for all azimuths, since $I$ is azimuthally invariant. Then we apply a stochastic inversion based on equation:

$$
Q(\phi)=G_{i s o}+G_{a n i} \cos ^{2}\left(\phi-\phi_{0}\right),
$$

where the input data, $Q(\phi)$, is previously obtained by the first inversion and $G_{i s o}, G_{a n i}$ and $\phi_{0}$ are parameters to be inverted. The initial model to $G_{i s o}$ can be done using the background velocity model and for $G_{a n i}$ and $\phi_{0}$ we consider initially null. Thus, it is possible to estimate the fracture orientation without any ambiguity.

\section{Isotropic and anisotropic parameters estimation}

We apply a stochastic seismic inversion to estimate the isotropic and anisotropic parameters. The stochastic inversion is set up in a Bayesian framework, estimating the posterior distribution function of the model parameters from a priori information and a likelihood function. For it, we apply a Metropolis-Hastings with annealing algorithm, which initially randomly perturbs an initial model based on prior information, i.e., changes the values of $\alpha, \beta$ and $\rho$ (isotropic estimation step) or the values of the anisotropic parameters (anisotropic estimation step). This perturbation can be done independently or we can link the parameters through empirical relationships obtained by nearby wells. Another alternative is to use a covariance matrix in the sampling step. The proposed model is accepted or not according the Metropolis criterion, if accepted, this model is used as new input to posterior perturbation and the process is repeated until achieve certain number of iterations, which theoretically will provide an approximation to desired posterior distribution.

Considering the equation (1) for the isotropy plane, i.e., $\phi \perp \phi_{0}$, we have:

$R_{P P}^{i s o}(i)$

$=\frac{1}{2} \frac{\Delta Z}{\bar{Z}}+\frac{1}{2}\left\{\frac{\Delta \alpha}{\bar{\alpha}}-\left(\frac{2 \bar{\beta}}{\bar{\alpha}}\right)^{2} \frac{\Delta G}{\bar{G}}\right\} \sin ^{2} i$

$+\frac{1}{2} \frac{\Delta \alpha}{\bar{\alpha}} \sin ^{2} i \tan ^{2} i$

which is the conventional AVO equation for isotropic case, depending only on isotropic parameters and incidence angles, as expected. As we already know the isotropyplane direction, it is possible to obtain the isotropic parameters by inversion using the seismic data in this azimuth.

For the symmetry-axis plane, $\phi \| \phi_{0}$, the equation (1) reduces to: 
$R_{P P}^{a n i}(i)$

$=\frac{1}{2} \frac{\Delta Z}{\bar{Z}}+\frac{1}{2}\left\{\frac{\Delta \alpha}{\bar{\alpha}}-\left(\frac{2 \bar{\beta}}{\bar{\alpha}}\right)^{2}\left(\frac{\Delta G}{\bar{G}}-2 \Delta \gamma\right)\right.$

$\left.+\Delta \delta^{(V)}\right\} \sin ^{2} i+\frac{1}{2}\left(\frac{\Delta \alpha}{\bar{\alpha}}+\Delta \epsilon^{(V)}\right) \sin ^{2} i \tan ^{2} i$,

which is dependent on the anisotropic Thomsen's parameters.

Applying the difference between the reflectivity for the isotropy plane and the symmetry-axis plane, we get:

$\Delta R_{P P}(i)=\frac{1}{2}\left\{-8\left(\frac{\bar{\beta}}{\bar{\alpha}}\right)^{2} \Delta \gamma-\Delta \delta^{(V)}\right\} \operatorname{sen}^{2}(i)-$

$\frac{1}{2}\left(\Delta \epsilon^{(V)}\right) \operatorname{sen}^{2}(i) \tan ^{2}(i)$,

This is an interesting procedure, since we considerably reduce the number of parameters maintaining only the anisotropic parameters, which are the focus of this step. We also make use of the Linear Slip Theory (LST) of Schoenberg and Sayers (1995) to restrict even more the number of parameters and to introduce some references to fracture properties. When the LST model is assumed, the Thomsen's parameters can be expressed in terms of normal and tangential weaknesses $\Delta_{N}$ and $\Delta_{T}$, respectively:

$$
\begin{gathered}
\epsilon^{(V)}=-2 g(1-g) \Delta_{N}, \\
\gamma^{(V)}=-\frac{1}{2} \Delta_{T}, \\
\delta^{(V)}=-2 g\left[(1-2 g) \Delta_{N}+\Delta_{T}\right],
\end{gathered}
$$

where $g$ is the square of $\beta$ and $\alpha$ ratio.

Considering the symmetry-axis plane and isotropy plane direction known we can estimate the anisotropic parameters, either Thomsen's parameters or normal and tangential weaknesses, from a stochastic inversion previously described.

\section{Poroelastic model - in-situ stress estimation}

Assuming that the rocks are constrained horizontally and undergoing elastic deformation, it is possible to derive expressions to estimate the magnitude of principal horizontal stresses based on simplified Hooke's law considering the LST compliance tensor (Gray et al., 2012). The principal effective horizontal stresses are given by the following equations:

$$
{\sigma^{\prime}}_{h}=\sigma_{V}^{\prime} \frac{v(1+v)}{1+E Z_{N}-v^{2}}
$$

and

$$
\sigma_{H}^{\prime}=\sigma_{V}^{\prime} v \frac{1+E Z_{N}+v}{1+E Z_{N}-v^{2}}
$$

where $\sigma_{h}^{\prime}$ is the minimum effective horizontal stress, $\sigma_{H}^{\prime}$ is the maximum effective horizontal stress, $\sigma_{V}^{\prime}$ is the effective vertical stress, $E$ is the Young's modulus, $v$ is the Poisson's ratio and $Z_{N}$ is the normal compliance. The effective term expresses the difference between the absolute stress and the pore pressure.

The minimum and maximum effective horizontal stresses are a function of $\sigma_{V}^{\prime}, E, v$ and $Z_{N}$. The first three parameters can be estimated by the output parameters provided by the isotropic stochastic inversion. The normal compliance is estimated by the equation (13), which $\Delta_{N}$ is the normal weakness, obtained in the anisotropic inversion step.

$$
Z_{N}=\frac{\Delta_{N}(1+v)(1-2 v)}{\left(1-\Delta_{N}\right) E(1-v)}
$$

\section{Results}

To verify the applicability of the proposed methodology we do an inversion test in a 1D synthetic data. The azimuthal synthetic gather (Fig. 2) is modeled using the convolutional model, whose reflectivity series is obtained from equation (1). The elastic parameters used are related to a well section with presence of natural fractures, which anisotropy is identified through advanced sonic methods and borehole image interpretation. The logs are not shown because confidentiality.

It has 5 traces for each source-receiver azimuth, with incidence angle $i \in\left(0^{\circ}, 40^{\circ}\right)$, azimuth $\phi=\left[25^{\circ}, 55^{\circ}, 85^{\circ}\right.$, $\left.115^{\circ}, 145^{\circ}, 175^{\circ}\right]$ and $\phi_{0}=145^{\circ}$. The wavelet used is a Ricker of $20 \mathrm{~Hz}$ central frequency.

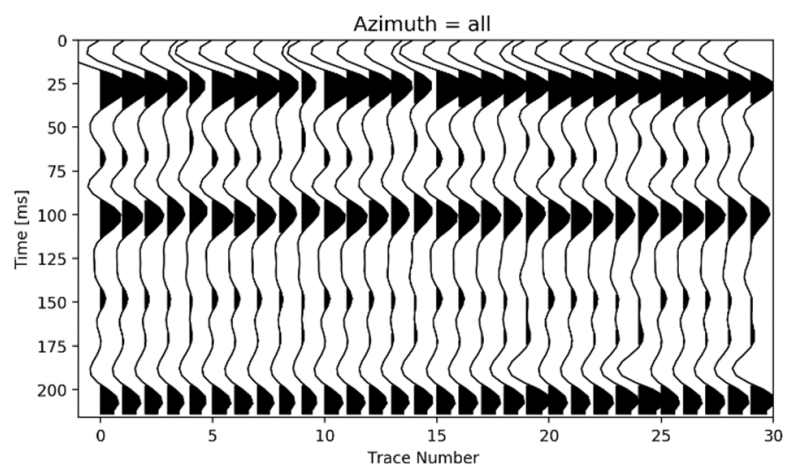

Figure 2: From left to right are: the azimuthal seismic response modeled for 6 azimuths $\left(25^{\circ}, 55^{\circ}, 85^{\circ}, 115^{\circ}\right.$, $145^{\circ}, 175^{\circ}$ ) with 5 traces each, e.g., the $25^{\circ}$ azimuth gather is composed by $0-4$ trace indexes, the $55^{\circ}$ for $5-9$ trace indexes, etc.

As defined previously, the first step in the methodology is to determine the fracture orientation applying a deterministic inversion procedure using equation (2) obtaining different values of gradient $Q$ to different azimuths. After that, it was possible to apply the stochastic inversion based on equation (4) and recovery the symmetry-axis plane. Subtracting $90^{\circ}$ degree to the symmetry-axis we obtain the fracture direction (isotropy plane). The Fig. 3 shows a histogram with the fracture 
direction values estimated. It is possible to see that the major quantity of the samples in the inversion analysis interval agree with the true parameter $\left(\sim 55^{\circ}\right)$ and no ambiguity exists.

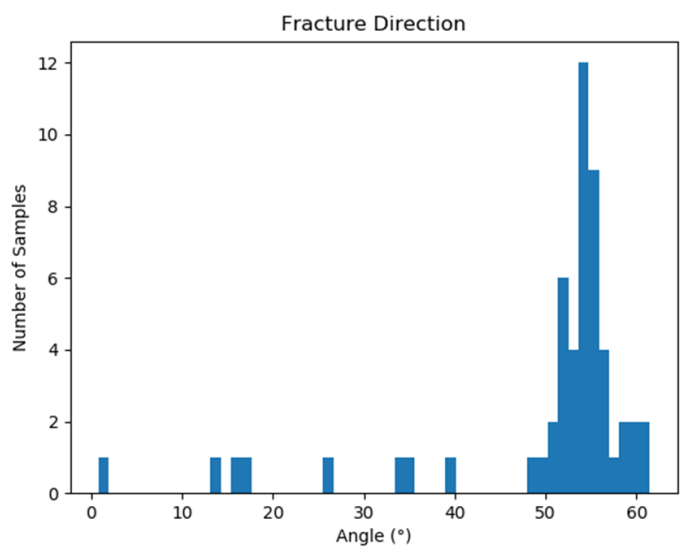

Figure 3: Histogram of the fracture direction estimated.

Knowing the direction of the symmetry-axis and isotropy plane, the next step is to estimate the isotropic and anisotropic parameters through stochastic inversion. In this work the isotropic estimation step was not done, since one of the main objectives is to estimate the anisotropic parameters using the difference between iso-aniso gathers, besides the isotropic inversion is a well-known approach.

The anisotropic estimation step consists in a stochastic seismic inversion, which the data used is obtained by subtraction of the isotropic gather, gather in the isotropyplane direction - azimuth equal $55^{\circ}$, and gather in the symmetry-axis plane - azimuth equal $145^{\circ}$, as shown in Fig. 4.

In this step we constrained our inversion by the LST physical model using equations (8)-(10), thus our forward model is in function of normal and tangential weaknesses, $\Delta_{N}$ and $\Delta_{T}$, respectively, reducing the number of parameters by one. As input model to stochastic inversion we consider null anisotropy and to the isotropic parameter $g$ we consider a constant. The inversion was processed until we reach a certain number of iterations, that in this case was approximately 5000, and we obtained the weaknesses as output (Fig. 5).

As seen by the red and green curves in Fig. 5, the interval of interest has similar values to $\Delta_{N}$ and $\Delta_{T}$, showing average values observed when the presence of dry cracks is dominant (Bakulin et al., 2000). We can differentiate a region with more anisotropy, sample 0 to 30 approximately, and a region which anisotropy is not so considerable, sample 30 to 50 . The results prevenient from inversion correlate in a satisfactory way with the true parameters, since it is possible to separate the different intervals described, more and less anisotropy, although some higher weakness are estimated in an interval with less anisotropy.

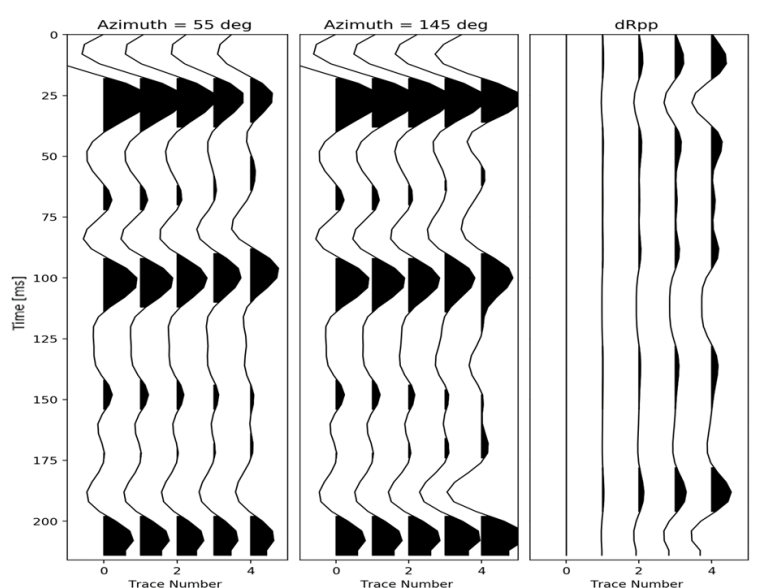

Figure 4: From left to right: isotropic angle gather, anisotropic angle gather, and difference between them, which is used as data input to anisotropic seismic inversion.

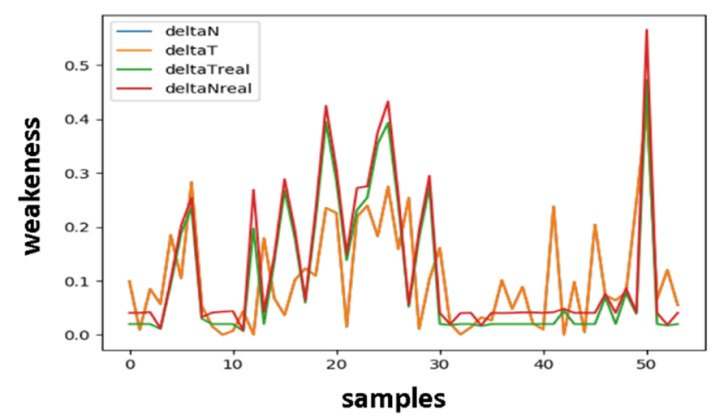

Figure 5: Normal and tangential weaknesses estimated by inversion compared with true parameters obtained by well logs.

To calculate the principal stresses for the inverted model we use the equations (11) and (12), where the parameter $Z_{N}$ is calculated by equation (13). The elastic moduli $E$ and $v$ were calculated using well log information, the vertical stress $\sigma_{V}$ is obtained by integrating logged density, and the pore pressure is estimated by Eaton's method (1975).

In Fig.6 was compared the horizontal in-situ stresses, obtained by processing well log data following an 1D MEM sequence (Plumb et al., 2000), with the horizontal stress curves estimated by stochastic inversion approach. The additional curves are: overburden $\sigma_{V}$ (integrated density) and pore pressure $P P$. We display all these curves in order to compare and validate the inversion results in function of this available data.

Comparing the modeled and inverted horizontal stress curves it is possible to see the good agreement between them, which the curve patterns are maintained. In the interval from 0.07 to 0.12 seconds we can see certain discrepancy between the inverted and modeled minimum horizontal stress, this was associated to the normal weakness parameter obtained in the anisotropic inversion, which exist a minimal difference from the real one. Although this discrepancy, the results are satisfactory and 


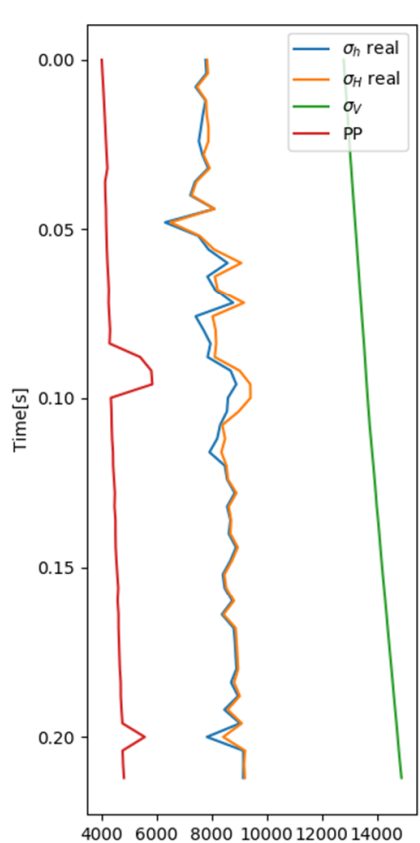

psi

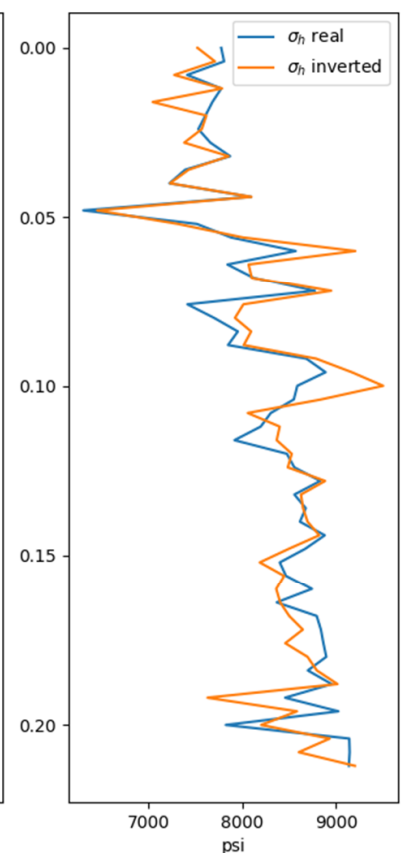

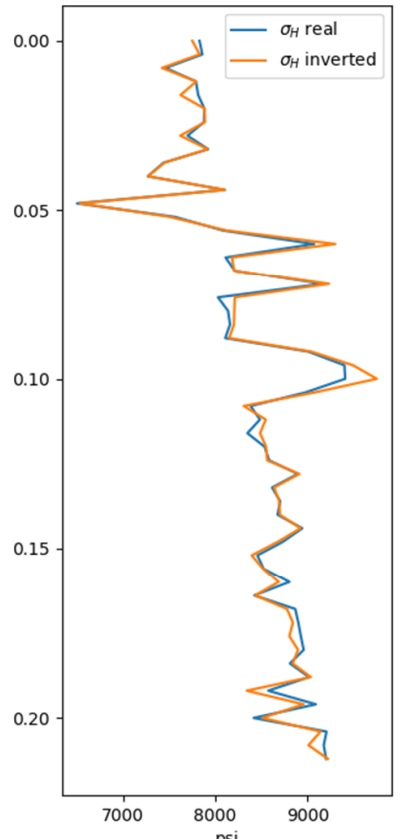

psi

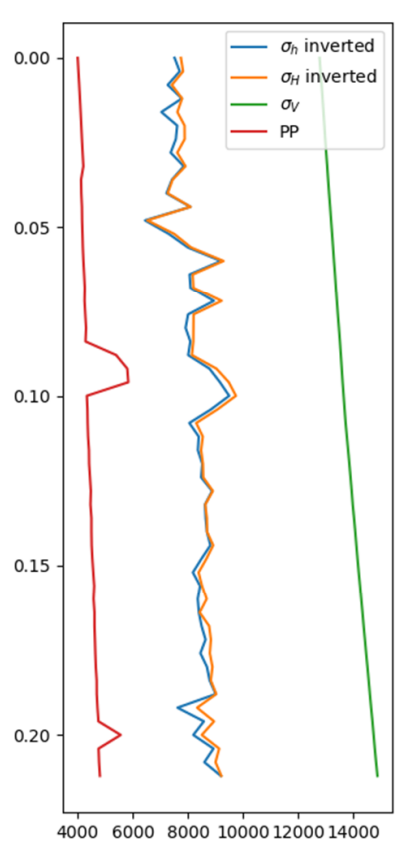

psi

Figure 6: From left to right: the stresses curves modeled using well log information; the comparison between real and inverted minimum horizontal stress; the comparison between real and inverted maximum horizontal stress; the stress inverted model containing the stresses curves obtained by inverted parameters.

gives us a good estimation of important parameters to reservoir characterization and development.

\section{Conclusions and recommendations}

We demonstrate a methodology for geomechanical properties and in-situ stress estimation using a stochastic seismic anisotropy sequential inversion. This approach is based on Rüger approximation for an HTI media and use the physical model LST to relate anisotropy and stress. The sequential methodology proved be applicable, which the first step, fracture orientation determination, through a deterministic inversion to different gradients and then stochastic inversion to fracture direction, was able to eliminate the ambiguity of $90^{\circ}$. Knowing the fracture direction it is possible to obtain the isotropic and anisotropic parameters.

The isotropic estimation is done through stochastic inversion, which can be used empirical relationships to restrict the problem. This step is well-known but essential during this methodology, since this result will provide the elastic parameters necessary to calculate the vertical stress, and the elastic moduli.

The anisotropic estimation is the key step, which the input data to inversion is the difference between the isotropic and anisotropic gather, being distinctive from other methods. This difference allows the inversion be only in function of the anisotropic parameters, providing a better estimation of them, since was already proved that in the inversion of all parameters together, the isotropic terms have more influence on the reflection coefficients. An important point is the necessity of large offsets, because the anisotropy effect is mainly contained in them.

At least, we estimate a reliable geomechanical properties and in-situ stresses, proving the applicability of the proposed methodology. As suggestion, we recommend to verify the efficiency of the methodology in a real azimuthal seismic data.

\section{Acknowledgments}

We thank ANP and Queiroz Galvão Exploration and Production for support this project.

\section{References}

Bakulin, A., Grechka, V., and Tsvankin, I., 2000, Estimation of fracture parameters from reflection seismic data - Part I: HTI model due to a single fracture set: Geophysics, 65, 1788-1802;

Delbecq, F., Downton, J. E., and Letizia, M., 2013, A mathfree look at azimuthal surface seismic techniques: CSEG Recorder, 38, 20-31;

Downton, J. and Gray, D., 2006, AVAZ parameter uncertainty estimation: SEG Expanded Abstracts, 234237;

Eaton, B. A., 1975, The equation for geopressure prediction from well logs: SPE 5544;

Gray, D., Anderson, P., Logel, J., Delbecq, F., Schmidt, D., and Schmid, R., 2012, Estimation of stress and 
geomechanical properties using 3D seismic data: First Break, 30, 59-68;

Mahmoudian, F., and Margrave, G. F., 2013, AVAZ inversion for fracture orientation and intensity - a physical modeling study. In 75th EAGE Conference \& Exhibition incorporating SPE EUROPEC;

Mavko, G., Mukerji, T., and Godfrey, N., 1995, Predicting stress-induced velocity anisotropy in rocks: Geophysics, 60, 1081-1087;

Ostrander, W. J., 1984, Plane-wave reflection coefficients for gas sands at non-normal angles of incidence: Geophysics, 49, 1637-1648,

Plumb, R., Edwards, S., Pidcock, G., Lee, D., and Stacey, B., 2000, The mechanical earth model concept and its application to high-risk well construction projects. In IADC/SPE Drilling Conference, New Orleans, Louisiana. Society of Petroleum Engineers, IADC/SPE 59128;

Richards, P. G., and Frasier, C. W., 1976, Scattering of elastic waves from depth-dependent inhomogeneities: Geophysics, 41, 441-458;

Rüger, A., 1997, P-wave reflection coefficients for transversely isotropic models with vertical and horizontal axis of symmetry: Geophysics, 62, 713-722;

Rüger, A., 1998, Variation of P-wave reflectivity with offset and azimuth in anisotropic media: Geophysics, 63, 935947 ;

Schoenberg, M., and Sayers, C. M., 1995, Seismic anisotropy of fractured rocks: Geophysics, 60, 204-211;

Shuey, R. T., 1985, A simplification of the Zoeppritz equations: Geophysics, 50, 609-614;

Thomsen, L., 1986, Weak elastic anisotropy: Geophysics, 51, 1954-1966;

Thomsen, L., 1993, Weak anisotropic reflections, in Offset dependent reflectivity, Castagna, J., and Backus, M., Eds: Soc. Expl. Geophys, 103-114;

Williams, M., and Jenner, E., 2002, Interpreting seismic data in the presence of azimuthal anisotropy; or azimuthal anisotropy in the presence of the seismic interpretation. The Leading Edge, 21, 771-774. 